\title{
Some New Discrete Gronwall-Bellman Type Inequalities with Three Independent Variables and Applications
}

\author{
Weihua Liu $\mathbb{D}^{1}$ and Haisong Huang $\mathbb{D}^{2}$ \\ ${ }^{1}$ College of Information and Management Science, Henan Agricultural University, Zhengzhou 450002, China \\ ${ }^{2}$ Faculty of General Education, Zhengzhou Technology and Business University, Zhengzhou 450026, China \\ Correspondence should be addressed to Weihua Liu; 969287779@qq.com
}

Received 3 May 2018; Accepted 3 July 2018; Published 1 August 2018

Academic Editor: Hugo Leiva

Copyright (c) 2018 Weihua Liu and Haisong Huang. This is an open access article distributed under the Creative Commons Attribution License, which permits unrestricted use, distribution, and reproduction in any medium, provided the original work is properly cited.

\begin{abstract}
Some new discrete Gronwall-Bellman type inequalities with three independent variables which generalize some existing results and can be used as handy tools in the study of qualitative and quantitative properties of solutions of certain classes of difference equation are presented. As applications, some difference equations with the initial boundary conditions are also considered.
\end{abstract}

\section{Introduction}

Finite difference inequalities which exhibit explicit bounds on unknown functions in general provide a very useful and important tool in the development of the theory of finite difference equations. Due to the fact that finite difference inequalities have been applied in various branches of finite difference equations, many such inequalities with in one or two variables have been established [1-4]. For example, Li $[5,6]$ investigated some new discrete inequalities in two independent variables which provide explicit bounds on unknown functions. Cheung [7-9] generalized some existing discrete Gronwall-Bellman-type inequalities to more general situations and applied them to study the boundedness, uniqueness, and continuous dependence of solutions of certain discrete boundary value problem for difference equations.

Wang [10-13] established some nonlinear retarded difference inequalities and gave some applications of the obtained inequalities to the estimation of finite difference equations. Pachpatte [14-16] obtained certain inequalities arising in the theory of differential equations and provided new estimates for these types of inequalities. Ma et al. [17-19] obtained some power nonlinear Volterra-Fredholm type discrete inequalities. However, to the best of the authors' knowledge, few papers are only published on discrete inequalities with three independent variables. Hussain et al. [20] discussed some generalized Volterra-Fredholm type nonlinear discrete inequalities involving four iterated infinite sums and applied these results to new explicit bounds for solutions of certain difference equations.

In 1979, Pachpatte and Singare [21] gave discrete Gronwall type inequalities in three independent variables as follows.

Theorem 1. Let $u(x, y, z)$ and $p(x, y, z)$ be real-valued nonnegative functions defined for $(x, y, z) \in \mathbb{N}_{0}^{3}\left(\mathbb{N}_{0}:=\{0,1\right.$, $2, \ldots\})$ for which the inequality

$$
\begin{aligned}
u(x, y, z) \leq & a(x)+b(y)+c(z) \\
& +\sum_{r=0}^{x-1} \sum_{s=0}^{y-1} \sum_{t=0}^{z-1} p(r, s, t) u(r, s, t),
\end{aligned}
$$

holds for $(x, y, z) \in \mathbb{N}_{0}^{3}$, where $a(x), b(y), c(z)>0$; $\Delta a(x), \Delta b(y), \Delta c(z)>0$ ( $\Delta$ is the operator defined by $\Delta u(n)=$ $u(n+1)-u(n))$ are real-valued functions defined on $\mathbb{N}_{0}$. Then

$$
\begin{aligned}
& u(x, y, z) \leq[a(0)+b(y)+c(z)] \\
& \quad \cdot \prod_{r=0}^{x-1}\left(1+\frac{\Delta a(x)}{a(r)+b(0)+c(z)}+\sum_{s=0}^{y-1} \sum_{t=0}^{z-1} p(r, s, t)\right),
\end{aligned}
$$

for $(x, y, z) \in \mathbb{N}_{0}^{3}$. 
In 1980, Singare and Pachpatte [22] established generalization of discrete Gronwall type inequalities in three independent variables in Theorem 1 in the following.

Theorem 2. Let $u(x, y, z)$ and $b(x, y, z)$ be real-valued nonnegative functions defined for $(x, y, z) \in \mathbb{N}_{0}^{3}$, and let $a(x, y, z)$ be positive nondecreasing in the three variables, defined for $(x, y, z) \in \mathbb{N}_{0}^{3}$ for which the inequality

$$
u(x, y, z) \leq a(x, y, z)+\sum_{r=0}^{x-1} \sum_{s=0}^{y-1} \sum_{t=0}^{z-1} b(r, s, t) u(r, s, t),
$$

holds for $(x, y, z) \in \mathbb{N}_{0}^{3}$. Then

$$
u(x, y, z) \leq a(x, y, z) \prod_{r=0}^{x-1}\left(1+\sum_{s=0}^{y-1} \sum_{t=0}^{z-1} p(r, s, t)\right)
$$

for $(x, y, z) \in \mathbb{N}_{0}^{3}$.

Motivated by the results mentioned above, the aim of this paper is to establish some new and more general discrete Gronwall-Bellman type inequalities involving functions of three independent variables, which generalize and extend some existing results in $[8,17-19,21,22]$ and can be readily used as handy and powerful tools in the analysis of certain classes of partial finite difference and sum-difference equations. We also give some applications to convey the importance of these new inequalities to study various properties of the solutions of a class of initial value problems involving difference equations.

\section{Main Results}

In this section, we present some new and more general discrete Gronwall-Bellman type inequalities in three independent variables which might be useful tools in the analysis of certain classes of partial finite difference and sum-difference equations.

In what follows, $I:=\left[x_{0}, X\right) \cap \mathbb{Z}, J:=\left[y_{0}, Y\right) \cap \mathbb{Z}$ and $K:=\left[z_{0}, Z\right) \cap \mathbb{Z}$ are three fixed lattices of integral points in $\mathbb{R}$, where $x_{0}, y_{0}, z_{0} \in \mathbb{Z}, X, Y, Z \in \mathbb{Z} \cup\{\infty\}$. Let $\Omega:=I \times J \times K \subset \mathbb{Z}^{3}, \mathbb{R}_{+}:=(0, \infty), \mathbb{R}_{0}:=[0, \infty)$ and $\Omega_{(r, s, t)}$ denote the sublattice $\left[l_{0}, r\right] \times\left[m_{0}, s\right] \times\left[n_{0}, t\right] \cap \Omega$ of $\Omega$ for any $(r, s, t) \in \Omega$. We also use the following notions of the operators $\Delta_{x} u(x, y, z):=u(x+1, y, z)-u(x, y, z)$, $\Delta_{y} u(x, y, z):=u(x, y+1, z)-u(x, y, z), \Delta_{z} u(x, y, z):=$ $u(x, y, z+1)-u(x, y, z)$, and $\Delta_{x y}^{2} u(x, y, z):=\Delta_{x} u(x, y+$ $1, z)-\Delta_{x} u(x, y, z)$, and $\Delta_{x y z}^{3} u(x, y, z):=\Delta_{x y}^{2} u(x, y, z+1)-$ $\Delta_{x y}^{2} u(x, y, z)$.

Theorem 3. Let $u, a, c$, and $f$ be real-valued nonnegative functions defined for $\Omega$ and $a$ and $c$ be nondecreasing in each variables. Suppose that $\varphi \in C\left(\mathbb{R}_{0}, \mathbb{R}_{0}\right)$ is a strictly increasing function with $\varphi(0)=0$ and $\psi(u)$ is a nondecreasing continuous function with $\psi(u)>0$ for $u \in \mathbb{R}_{+}$. If

$$
\begin{aligned}
& \varphi(u(x, y, z)) \\
& \quad \leq a(x, y, z) \\
& \quad+c(x, y, z) \sum_{r=x_{0}}^{x-1} \sum_{s=y_{0}}^{y-1} \sum_{t=z_{0}}^{z-1} f(r, s, t) \psi(u(r, s, t)),
\end{aligned}
$$

for all $(x, y, z) \in \Omega$, then

$$
\begin{aligned}
& u(x, y, z) \leq \varphi^{-1}\left\{\Phi^{-1}(\Phi(a(x, y, z))\right. \\
& \left.\left.+c(x, y, z) \sum_{r=x_{0}}^{x-1} \sum_{s=y_{0}}^{y-1} \sum_{t=z_{0}}^{z-1} f(r, s, t)\right)\right\},
\end{aligned}
$$

for all $(x, y, z) \in\left[x_{0}, x_{1}\right] \times\left[y_{0}, y_{1}\right] \times\left[z_{0}, z_{1}\right]$, where

$$
\Phi(r)=\int_{r_{0}}^{r} \frac{d s}{\psi\left[\varphi^{-1}(s)\right]}, \quad r \geq r_{0}>0,
$$

$\varphi^{-1}, \Phi^{-1}$ denote the inverse functions of $\varphi$, $\Phi$ and $\left(x_{1}, y_{1}, z_{1}\right) \in$ $\Omega$ is chosen so that

$$
\begin{aligned}
& \left(\Phi(a(x, y, z))+c(x, y, z) \sum_{r=x_{0}}^{x-1} \sum_{s=y_{0}}^{y-1} \sum_{t=z_{0}}^{z-1} f(r, s, t)\right) \\
& \quad \in \operatorname{dom}\left(\Phi^{-1}\right) .
\end{aligned}
$$

Proof. It suffices to consider the case $a(x, y, z)>0$, since the case $a(x, y, z)=0$ can be arrived at by continuity argument. Let us first assume that $a(x, y, z)>0$. Fixing any numbers $\bar{x}$, $\bar{y}$, and $\bar{z}$ with $x_{0} \leq x \leq \bar{x}, y_{0} \leq y \leq \bar{y}$ and $z_{0} \leq z \leq \bar{z}$, define a positive function $\omega(x, y, z)$ by

$$
\begin{aligned}
& \omega(x, y, z) \\
& \quad:=a(\bar{x}, \bar{y}, \bar{z}) \\
& \quad+c(\bar{x}, \bar{y}, \bar{z}) \sum_{r=x_{0}}^{x-1} \sum_{s=y_{0}}^{y-1} \sum_{t=z_{0}}^{z-1} f(r, s, t) \psi(u(r, s, t)) .
\end{aligned}
$$

Then $\omega(x, y, z)>0, \omega\left(x_{0}, y, z\right)=\omega\left(x, y_{0}, z\right)=\omega\left(x, y, z_{0}\right)=$ $a(\bar{x}, \bar{y}, \bar{z})$ and (5) can restated by

$$
u(x, y, z) \leq \varphi^{-1}[\omega(x, y, z)] \quad \text { on } \Omega_{(\bar{x}, \bar{y}, \bar{z})} .
$$

It is easy observe that $\omega(x, y, z)$ is a nondecreasing function in each variables defined for $\Omega_{(\bar{x}, \bar{y}, \bar{z})}$ and

$$
\begin{gathered}
\Delta_{x} \omega(x, y, z)=\omega(x+1, y, z)-\omega(x, y, z) \\
=c(\bar{x}, \bar{y}, \bar{z}) \sum_{s=y_{0}}^{y-1} \sum_{t=z_{0}}^{z-1} f(x, s, t) \psi(u(x, s, t)), \\
\Delta_{x y}^{2} \omega(x, y, z)=\Delta_{x} \omega(x, y+1, z)-\Delta_{x} \omega(x, y, z) \\
=c(\bar{x}, \bar{y}, \bar{z}) \sum_{t=z_{0}}^{z-1} f(x, y, t) \psi(u(x, y, t)) .
\end{gathered}
$$


From (11) we have

$$
\begin{aligned}
& \Delta_{x y}^{2} \omega(x, y, z+1)-\Delta_{x y}^{2} \omega(x, y, z) \\
& \quad=c(\bar{x}, \bar{y}, \bar{z}) f(x, y, z) \psi(u(x, y, z)) \\
& \quad \leq c(\bar{x}, \bar{y}, \bar{z}) f(x, y, z) \psi\left[\varphi^{-1}(\omega(x, y, z))\right] .
\end{aligned}
$$

From (12) we observe that

$$
\begin{gathered}
\frac{\Delta_{x y}^{2} \omega(x, y, z+1)-\Delta_{x y}^{2} \omega(x, y, z)}{\psi\left[\varphi^{-1}(\omega(x, y, z))\right]} \\
\leq c(\bar{x}, \bar{y}, \bar{z}) f(x, y, z) .
\end{gathered}
$$

From (13) we obtain

$$
\begin{aligned}
& \frac{\Delta_{x y}^{2} \omega(x, y, z+1)}{\psi\left[\varphi^{-1}(\omega(x, y, z+1))\right]}-\frac{\Delta_{x y}^{2} \omega(x, y, z)}{\psi\left[\varphi^{-1}(\omega(x, y, z))\right]} \\
& \quad \leq c(\bar{x}, \bar{y}, \bar{z}) f(x, y, z) .
\end{aligned}
$$

Now keeping $x, y$ fixed in (14), setting $z=t$, and substituting $t=z_{0}, z_{0}+1, \ldots, z-1$ in (14) we obtain the estimate for $\Delta_{x y}^{2} \omega(x, y, z) / \psi\left[\varphi^{-1}(\omega(x, y, z))\right]$ such that

$$
\frac{\Delta_{x y}^{2} \omega(x, y, z)}{\psi\left[\varphi^{-1}(\omega(x, y, z))\right]} \leq c(\bar{x}, \bar{y}, \bar{z}) \sum_{t=z_{0}}^{z-1} f(x, y, t) .
$$

From (15) we observe that

$$
\begin{aligned}
& \frac{\Delta_{x} \omega(x, y+1, z)}{\psi\left[\varphi^{-1}(\omega(x, y+1, z))\right]}-\frac{\Delta_{x} \omega(x, y, z)}{\psi\left[\varphi^{-1}(\omega(x, y, z))\right]} \\
& \quad \leq c(\bar{x}, \bar{y}, \bar{z}) \sum_{t=z_{0}}^{z-1} f(x, y, t) .
\end{aligned}
$$

Keeping $x, z$ fixed in (16), setting $y=s$, and substituting $s=y_{0}, y_{0}+1, \ldots, y-1$ in (16) we obtain the estimate for $\Delta_{x} \omega(x, y, z) / \psi\left[\varphi^{-1}(\omega(x, y, z))\right]$ such that

$$
\frac{\Delta_{x} \omega(x, y, z)}{\psi\left[\varphi^{-1}(\omega(x, y, z))\right]} \leq c(\bar{x}, \bar{y}, \bar{z}) \sum_{s=y_{0}}^{y-1} \sum_{t=z_{0}}^{z-1} f(x, s, t) .
$$

From the definition of $\Phi$ and (17) we obtain

$$
\begin{aligned}
\Phi & (\omega(x+1, y, z))-\Phi(\omega(x, y, z)) \\
& =\int_{\omega(x, y, z)}^{\omega(x+1, y, z)} \frac{d s}{\psi\left[\varphi^{-1}(s)\right]} \leq \frac{\Delta_{x} \omega(x, y, z)}{\psi\left[\varphi^{-1}(\omega(x, y, z))\right]} \\
& \leq c(\bar{x}, \bar{y}, \bar{z}) \sum_{s=y_{0}}^{y-1} \sum_{t=z_{0}}^{z-1} f(x, s, t) .
\end{aligned}
$$

Again keeping $y, z$ fixed in (18), setting $x=r$, and substituting $r=x_{0}, x_{0}+1, \ldots, x-1$ in (18), we obtain

$$
\begin{aligned}
\Phi(\omega(x, y, z)) \leq & \Phi(a(\bar{x}, \bar{y}, \bar{z})) \\
& +c(\bar{x}, \bar{y}, \bar{z}) \sum_{r=x_{0}}^{x-1} \sum_{s=y_{0}}^{y-1} \sum_{t=z_{0}}^{z-1} f(x, s, t) .
\end{aligned}
$$

Now using inequality (19) in (10), we get

$$
\begin{aligned}
& u(x, y, z) \leq \varphi^{-1}\left\{\Phi^{-1}(\Phi(a(\bar{x}, \bar{y}, \bar{z}))\right. \\
& \left.\left.\quad+c(\bar{x}, \bar{y}, \bar{z}) \sum_{r=x_{0}}^{x-1} \sum_{s=y_{0}}^{y-1} \sum_{t=z_{0}}^{z-1} f(r, s, t)\right)\right\} .
\end{aligned}
$$

Taking $x=\bar{x}, y=\bar{x}$, and $z=\bar{z}$ in inequality (20), since $\bar{x}, \bar{y}$, and $\bar{z}$ are arbitrary, we get the required inequality.

Corollary 4. Let $u, a, c, f$, and $\varphi$ be as defined in Theorem 3. If

$$
\begin{aligned}
& \varphi(u(x, y, z)) \\
& \leq a(x, y, z) \\
& \quad+c(x, y, z) \sum_{r=x_{0}}^{x-1} \sum_{s=y_{0}}^{y-1} \sum_{t=z_{0}}^{z-1} f(r, s, t) \varphi(u(r, s, t)),
\end{aligned}
$$

for all $(x, y, z) \in \Omega$, then

$$
\begin{aligned}
& u(x, y, z) \leq \varphi^{-1}\{a(x, y, z) \\
& \left.\cdot \exp \left(c(x, y, z) \sum_{r=x_{0}}^{x-1} \sum_{s=y_{0}}^{y-1} \sum_{t=z_{0}}^{z-1} f(r, s, t)\right)\right\},
\end{aligned}
$$

for all $(x, y, z) \in \Omega$.

Proof. Suppose first that $a(x, y, z)>0$. Taking $\psi=\varphi$, we have

$$
\Phi(r)=\int_{r_{0}}^{r} \frac{d s}{\varphi\left[\varphi^{-1}(s)\right]}=\int_{r_{0}}^{r} \frac{d s}{s}=\ln \frac{r}{r_{0}}, \quad r \geq r_{0}>0,
$$

and so $\Phi^{-1}(r)=r_{0} \exp r$, in particular, it is defined everywhere on $\mathbb{R}$. Hence, by Theorem 3 ,

$$
\begin{aligned}
& u(x, y, z) \leq \varphi^{-1}\left\{r _ { 0 } \operatorname { e x p } \left(\ln \frac{a(x, y, z)}{r_{0}}\right.\right. \\
& \left.\left.\quad+c(x, y, z) \sum_{r=x_{0}}^{x-1} \sum_{s=y_{0}}^{y-1} \sum_{t=z_{0}}^{z-1} f(r, s, t)\right)\right\}=\varphi^{-1}\{a(x, \\
& \left.y, z) \exp \left(c(x, y, z) \sum_{r=x_{0}}^{x-1} \sum_{s=y_{0}}^{y-1} \sum_{t=z_{0}}^{z-1} f(r, s, t)\right)\right\}
\end{aligned}
$$

for all $(x, y, z) \in \Omega$. Finally, by continuity, this should also hold for the case $c=0$. 
Theorem 5. Let $u, a, c$, and $f_{i}, g_{i}(i=1,2, \ldots, m)$ be real-valued nonnegative functions defined for $\Omega$ and $a$ and $c$ be nondecreasing in each variables. Suppose that $\varphi, \phi \in$
$C\left(\mathbb{R}_{0}, \mathbb{R}_{0}\right)$ are strictly increasing functions with $\varphi(0)=0$ and $\phi(0)=0$ and $\psi_{i}(u)$ is a nondecreasing continuous function with $\psi_{i}(u)>0(i=1,2, \ldots, m)$ for $u \in \mathbb{R}_{+}$. If

$$
\varphi(u(x, y, z)) \leq a(x, y, z)+c(x, y, z)\left\{\sum_{r=x_{0}}^{x-1} \sum_{s=y_{0}}^{y-1} \sum_{t=z_{0}}^{z-1} \phi(u(r, s, t)) \sum_{i=1}^{m}\left[f_{i}(r, s, t) \psi_{i}(u(r, s, t))+g_{i}(r, s, t)\right]\right\},
$$

for all $(x, y, z) \in \Omega$, let $\psi=\max _{1 \leq i \leq m}\left\{\psi_{i}\right\}$, then

$$
\begin{array}{r}
u(x, y, z) \leq \varphi^{-1}\left\{G ^ { - 1 } \left[\Phi ^ { - 1 } \left(\Phi\left(k\left(x_{0}, y, z\right)\right)\right.\right.\right. \\
\left.\left.\left.+c(x, y, z) \sum_{r=x_{0}}^{x-1} \sum_{s=y_{0}}^{y-1} \sum_{t=z_{0}}^{z-1} \sum_{i=1}^{m} f_{i}(x, y, z)\right)\right]\right\},
\end{array}
$$

for all $(x, y, z) \in\left[x_{0}, x_{1}\right] \times\left[y_{0}, y_{1}\right] \times\left[z_{0}, z_{1}\right]$, where

$$
\begin{aligned}
k\left(x_{0}, y, z\right)= & G(a(x, y, z)) \\
& +c(x, y, z) \sum_{r=x_{0}}^{x-1} \sum_{s=y_{0}}^{y-1} \sum_{t=z_{0}}^{z-1} \sum_{i=1}^{m} g_{i}(x, y, z),
\end{aligned}
$$

$$
\begin{aligned}
& G(r)=\int_{r_{0}}^{r} \frac{d s}{\phi\left[\varphi^{-1}(s)\right]}, \\
& \Phi(r)=\int_{r_{0}}^{r} \frac{d s}{\psi\left[\varphi^{-1}\left(G^{-1}(s)\right)\right]},
\end{aligned}
$$$$
r \geq r_{0}>0,
$$

$\varphi^{-1}, G^{-1}, \Phi^{-1}$ denote the inverse functions of $\varphi, G, \Phi$ and $\left(x_{1}, y_{1}, z_{1}\right) \in \Omega$ is chosen so that

$$
\begin{aligned}
& \left(\Phi(a(x, y, z))+c(x, y, z) \sum_{r=x_{0}}^{x-1} \sum_{s=y_{0}}^{y-1} \sum_{t=z_{0}}^{z-1} f(r, s, t)\right) \\
& \in \operatorname{dom}\left(\Phi^{-1}\right) .
\end{aligned}
$$

Proof. It suffices to consider the case $a(x, y, z)>0$, since the case $a(x, y, z)=0$ can be arrived at by continuity argument. Let us first assume that $a(x, y, z)>0$. Fixing any numbers $\bar{x}$, $\bar{y}$, and $\bar{z}$ with $x_{0} \leq x \leq \bar{x}, y_{0} \leq y \leq \bar{y}$ and $z_{0} \leq z \leq \bar{z}$, define a positive function $\omega(x, y, z)$ by

$$
\omega(x, y, z):=a(\bar{x}, \bar{y}, \bar{z})+c(\bar{x}, \bar{y}, \bar{z})\left\{\sum_{r=x_{0}}^{x-1} \sum_{s=y_{0}}^{y-1} \sum_{t=z_{0}}^{z-1} \phi(u(r, s, t)) \sum_{i=1}^{m}\left[f_{i}(r, s, t) \psi_{i}(u(r, s, t))+g_{i}(r, s, t)\right]\right\} .
$$

Then $\omega(x, y, z)>0, \omega\left(x_{0}, y, z\right)=\omega\left(x, y_{0}, z\right)=\omega\left(x, y, z_{0}\right)=$ $a(\bar{x}, \bar{y}, \bar{z})$ and (5) can restated by

$$
u(x, y, z) \leq \varphi^{-1}[\omega(x, y, z)] \quad \text { on } \Omega_{(\bar{x}, \bar{y}, \bar{z})} .
$$

It is easy observe that $\omega(x, y, z)$ is a nondecreasing function in each variable defined for $\Omega_{(\bar{x}, \bar{y}, \bar{z})}$ and

$$
\begin{aligned}
& \Delta_{x} \omega(x, y, z)=\omega(x+1, y, z)-\omega(x, y, z) \\
& \quad c(\bar{x}, \bar{y}, \bar{z}) \sum_{s=y_{0}}^{y-1} \sum_{t=z_{0}}^{z-1} \phi(u(x, s, t)) \\
& \cdot \sum_{i=1}^{m}\left[f_{i}(x, s, t) \psi_{i}(u(x, s, t))+g_{i}(x, s, t)\right],
\end{aligned}
$$

$$
\begin{gathered}
\Delta_{x y}^{2} \omega(x, y, z)=\Delta_{x} \omega(x, y+1, z)-\Delta_{x} \omega(x, y, z) \\
=c(\bar{x}, \bar{y}, \bar{z}) \sum_{t=z_{0}}^{z-1} \phi(u(x, y, t)) \\
\cdot \sum_{i=1}^{m}\left[f_{i}(x, y, t) \psi_{i}(u(x, y, t))+g_{i}(x, y, t)\right] .
\end{gathered}
$$

From (31) we have

$$
\begin{gathered}
\Delta_{x y}^{2} \omega(x, y, z+1)-\Delta_{x y}^{2} \omega(x, y, z)=c(\bar{x}, \bar{y}, \bar{z}) \\
\cdot \phi(u(x, y, z)) \sum_{i=1}^{m}\left[f_{i}(x, y, z) \psi_{i}(u(x, y, z))\right. \\
\left.+g_{i}(x, y, z)\right] \leq c(\bar{x}, \bar{y}, \bar{z}) \phi\left[\varphi^{-1}(\omega(x, y, z))\right]
\end{gathered}
$$




$$
\begin{aligned}
& \cdot \sum_{i=1}^{m}\left[f_{i}(x, y, z) \psi_{i}\left(\varphi^{-1}(\omega(x, y, z))\right)\right. \\
& \left.+g_{i}(x, y, z)\right] .
\end{aligned}
$$

From (32) we observe that

$$
\begin{aligned}
& \frac{\Delta_{x y}^{2} \omega(x, y, z+1)-\Delta_{x y}^{2} \omega(x, y, z)}{\phi\left[\varphi^{-1}(\omega(x, y, z))\right]} \leq c(\bar{x}, \bar{y}, \bar{z}) \\
& \cdot \sum_{i=1}^{m}\left[f_{i}(x, y, z) \psi_{i}\left(\varphi^{-1}(\omega(x, y, z))\right)\right. \\
& \left.\quad+g_{i}(x, y, z)\right] .
\end{aligned}
$$

From (33) we obtain

$$
\begin{aligned}
& \frac{\Delta_{x y}^{2} \omega(x, y, z+1)}{\phi\left[\varphi^{-1}(\omega(x, y, z+1))\right]}-\frac{\Delta_{x y}^{2} \omega(x, y, z)}{\phi\left[\varphi^{-1}(\omega(x, y, z))\right]} \\
& \quad \leq c(\bar{x}, \bar{y}, \bar{z}) \sum_{i=1}^{m}\left[f_{i}(x, y, z) \psi_{i}\left(\varphi^{-1}(\omega(x, y, z))\right)\right. \\
& \left.\quad+g_{i}(x, y, z)\right]
\end{aligned}
$$

$$
G(\omega(x, y, z)) \leq G(a(\bar{x}, \bar{y}, \bar{z}))+c(\bar{x}, \bar{y}, \bar{z}) \sum_{r=x_{0}}^{x-1} \sum_{s=y_{0}}^{y-1} \sum_{t=z_{0}}^{z-1} \sum_{i=1}^{m}\left[f_{i}(x, y, z) \psi_{i}\left(\varphi^{-1}(\omega(x, y, z))\right)+g_{i}(x, y, z)\right]
$$

Now, define function $k(x, y, z)$ by

$$
\begin{aligned}
& k(x, y, z):=G(a(\bar{x}, \bar{y}, \bar{z}))+c(\bar{x}, \bar{y}, \bar{z}) \\
& \quad \cdot \sum_{r=x_{0}}^{\bar{x}-1} \sum_{s=y_{0}} \sum_{t=z_{0}}^{\bar{y}-1} \sum_{i=1}^{m} g_{i}(x, y, z)+c(\bar{x}, \bar{y}, \bar{z}) \\
& \quad \cdot \sum_{r=x_{0}}^{x-1} \sum_{s=y_{0}}^{y-1} \sum_{t=z_{0}}^{z-1} \sum_{i=1}^{m} f_{i}(x, y, z) \psi_{i}\left(\varphi^{-1}(\omega(x, y, z))\right) .
\end{aligned}
$$

Then $k\left(x_{0}, y, z\right)=G(a(\bar{x}, \bar{y}, \bar{z}))+c(\bar{x}, \bar{y}$, $\bar{z}) \sum_{r=x_{0}}^{\bar{x}-1} \sum_{s=y_{0}}^{\bar{y}-1} \sum_{t=z_{0}}^{\bar{z}-1} \sum_{i=1}^{m} g_{i}(x, y, z)$ and (35) can be restated as

$$
\omega(x, y, z) \leq G^{-1}[k(x, y, z)] \quad \text { on } \Omega_{(\bar{x}, \bar{y}, \bar{z})} .
$$

It is easy observe that $k(x, y, z)$ is a nondecreasing function in each variable defined for $\Omega_{(\bar{x}, \bar{y}, \bar{z})}$ and

$$
\begin{aligned}
& \Delta_{x} k(x, y, z)=k(x+1, y, z)-k(x, y, z) \\
& =c(\bar{x}, \bar{y}, \bar{z}) \sum_{s=y_{0}}^{y-1} \sum_{t=z_{0}}^{z-1} \sum_{i=1}^{m} f_{i}(x, s, t) \psi_{i}(u(x, s, t)), \\
& \Delta_{x y}^{2} k(x, y, z)=\Delta_{x} k(x, y+1, z)-\Delta_{x} k(x, y, z) \\
& =c(\bar{x}, \bar{y}, \bar{z}) \sum_{t=z_{0}}^{z-1} \sum_{i=1}^{m} f_{i}(x, y, t) \psi_{i}(u(x, y, t)) .
\end{aligned}
$$

From (38) we have

$$
\begin{gathered}
\Delta_{x y}^{2} k(x, y, z+1)-\Delta_{x y}^{2} k(x, y, z)=c(\bar{x}, \bar{y}, \bar{z}) \\
\cdot \sum_{i=1}^{m} f_{i}(x, y, z) \psi_{i}(u(x, y, z)) \leq c(\bar{x}, \bar{y}, \bar{z}) \\
\cdot \psi\left[\varphi^{-1}\left(G^{-1}(k(x, y, z))\right)\right] \sum_{i=1}^{m} f_{i}(x, y, z) .
\end{gathered}
$$

From (39) we observe that

$$
\begin{gathered}
\frac{\Delta_{x y}^{2} k(x, y, z+1)-\Delta_{x y}^{2} k(x, y, z)}{\psi\left[\varphi^{-1}\left(G^{-1}(k(x, y, z))\right)\right]} \\
\leq c(\bar{x}, \bar{y}, \bar{z}) \sum_{i=1}^{m} f_{i}(x, y, z) .
\end{gathered}
$$

From (40) we obtain

$$
\begin{gathered}
\frac{\Delta_{x y}^{2} k(x, y, z+1)}{\psi\left[\varphi^{-1}\left(G^{-1}(k(x, y, z+1))\right)\right]} \\
-\frac{\Delta_{x y}^{2} k(x, y, z)}{\psi\left[\varphi^{-1}\left(G^{-1}(k(x, y, z))\right)\right]} \\
\leq c(\bar{x}, \bar{y}, \bar{z}) \sum_{i=1}^{m} f_{i}(x, y, z) .
\end{gathered}
$$

Along the lines of proof of Theorem 3, we obtain

$$
\begin{aligned}
& \Phi(k(x, y, z)) \\
& \leq \Phi\left(k\left(x_{0}, y, z\right)\right) \\
& \quad+c(\bar{x}, \bar{y}, \bar{z}) \sum_{r=x_{0}}^{x-1} \sum_{s=y_{0}}^{y-1} \sum_{t=z_{0}}^{z-1} \sum_{i=1}^{m} f_{i}(x, y, z) .
\end{aligned}
$$


Now using inequalities (37) and (42) in (30), we get

$$
\begin{aligned}
& u(x, y, z) \leq \varphi^{-1}\left\{G ^ { - 1 } \left[\Phi ^ { - 1 } \left(\Phi\left(k\left(x_{0}, y, z\right)\right)\right.\right.\right. \\
& \left.\left.\left.+c(\bar{x}, \bar{y}, \bar{z}) \sum_{r=x_{0}}^{x-1} \sum_{s=y_{0}}^{y-1} \sum_{t=z_{0}}^{z-1} \sum_{i=1}^{m} f_{i}(x, y, z)\right)\right]\right\} .
\end{aligned}
$$

Taking $x=\bar{x}, y=\bar{x}$, and $z=\bar{z}$ in inequality (43), since $\bar{x}, \bar{y}$, and $\bar{z}$ are arbitrary, we get the required inequality.

Corollary 6. Let $u, a, c, \varphi, \psi$ and $f_{i}, g_{i}, \psi_{i}(i=1,2, \ldots, m)$ be as defined in Theorem 5. If

$$
\varphi(u(x, y, z)) \leq a(x, y, z)+c(x, y, z)\left\{\sum_{r=x_{0}}^{x-1} \sum_{s=y_{0}}^{y-1} \sum_{t=z_{0}}^{z-1} \varphi(u(r, s, t)) \sum_{i=1}^{m}\left[f_{i}(r, s, t) \psi_{i}(u(r, s, t))+g_{i}(r, s, t)\right]\right\}
$$

for all $(x, y, z) \in \Omega$, let $\psi=\max _{1 \leq i \leq m}\left\{\psi_{i}\right\}$, then

$$
\begin{aligned}
& u(x, y, z) \leq \varphi^{-1}\{a(x, y, z) \\
& \cdot \exp \left[\Phi _ { 1 } ^ { - 1 } \left(\Phi_{1}\left(k_{1}\left(x_{0}, y, z\right)\right)\right.\right. \\
& \left.\left.\left.\quad+c(x, y, z) \sum_{r=x_{0}}^{x-1} \sum_{s=y_{0}}^{y-1} \sum_{t=z_{0}}^{z-1} \sum_{i=1}^{m} f_{i}(x, y, z)\right)\right]\right\},
\end{aligned}
$$

for all $(x, y, z) \in\left[x_{0}, x_{1}\right] \times\left[y_{0}, y_{1}\right] \times\left[z_{0}, z_{1}\right]$, where

$$
\begin{gathered}
k_{1}\left(x_{0}, y, z\right)=c(x, y, z) \sum_{r=x_{0}}^{x-1} \sum_{s=y_{0}}^{y-1} \sum_{t=z_{0}}^{z-1} \sum_{i=1}^{m} g_{i}(x, y, z), \\
\Phi_{1}(r)=\int_{r_{0}}^{r} \frac{d s}{\psi\left[\varphi^{-1}\left(a e^{s}\right)\right]}, \quad r \geq r_{0}>0,
\end{gathered}
$$

where $\varphi^{-1}, \Phi_{1}^{-1}$ denote the inverse functions of $\varphi, \Phi_{1}$ and $\left(x_{1}, y_{1}, z_{1}\right) \in \Omega$ is chosen so that

$$
\begin{aligned}
& \left(\Phi_{1}\left(k_{1}\left(x_{0}, y, z\right)\right)\right. \\
& \left.+c(x, y, z) \sum_{r=x_{0}}^{x-1} \sum_{s=y_{0}}^{y-1} \sum_{t=z_{0}}^{z-1} \sum_{i=1}^{m} f_{i}(x, y, z)\right) \\
& \quad \in \operatorname{dom}\left(\Phi_{1}^{-1}\right) .
\end{aligned}
$$

Proof. It suffices to consider the case $a(x, y, z)>0$, since the case $a(x, y, z)=0$ can be arrived at by continuity argument. Let us first assume that $a(x, y, z)>0$. Fixing any numbers $\bar{x}$, $\bar{y}$, and $\bar{z}$ with $x_{0} \leq x \leq \bar{x}, y_{0} \leq y \leq \bar{y}$, and $z_{0} \leq z \leq \bar{z}$, define a positive function $\omega(x, y, z)$ by

$$
\omega(x, y, z):=a(\bar{x}, \bar{y}, \bar{z})+c(\bar{x}, \bar{y}, \bar{z})\left\{\sum_{r=x_{0}}^{x-1} \sum_{s=y_{0}}^{y-1} \sum_{t=z_{0}}^{z-1} \varphi(u(r, s, t)) \sum_{i=1}^{m}\left[f_{i}(r, s, t) \psi_{i}(u(r, s, t))+g_{i}(r, s, t)\right]\right\}
$$

Then $\omega(x, y, z)>0, \omega\left(x_{0}, y, z\right)=\omega\left(x, y_{0}, z\right)=\omega\left(x, y, z_{0}\right)=$ $a(\bar{x}, \bar{y}, \bar{z})$ and $(44)$ can restated by

$$
u(x, y, z) \leq \varphi^{-1}[\omega(x, y, z)] \quad \text { on } \Omega_{(\bar{x}, \bar{y}, \bar{z})} .
$$

Along the lines of proof of Theorem 5, we obtain

$$
\frac{\Delta_{x y}^{2} \omega(x, y, z+1)-\Delta_{x y}^{2} \omega(x, y, z)}{\omega(x, y, z)} \leq c(\bar{x}, \bar{y}, \bar{z})
$$

$$
\begin{aligned}
& \cdot \sum_{i=1}^{m}\left[f_{i}(x, y, z) \psi_{i}\left(\varphi^{-1}(\omega(x, y, z))\right)\right. \\
& \left.+g_{i}(x, y, z)\right] .
\end{aligned}
$$

From the above inequality and Theorem 3, $G(r)=$ $\int_{r_{0}}^{r}\left(d s / \varphi\left[\varphi^{-1}(s)\right]\right)=\int_{r_{0}}^{r}(d s / s)=\ln \left(r / r_{0}\right), r \geq r_{0}>0$, we observe that

$$
\ln (\omega(x, y, z)) \leq \ln (a(\bar{x}, \bar{y}, \bar{z}))+c(\bar{x}, \bar{y}, \bar{z}) \sum_{r=x_{0}}^{x-1} \sum_{s=y_{0}}^{y-1} \sum_{t=z_{0}}^{z-1} \sum_{i=1}^{m}\left[f_{i}(x, y, z) \psi_{i}\left(\varphi^{-1}(\omega(x, y, z))\right)+g_{i}(x, y, z)\right]
$$


Now, define a function $k_{1}(x, y, z)$ by

$$
\begin{aligned}
& k_{1}(x, y, z):=c(\bar{x}, \bar{y}, \bar{z}) \sum_{r=x_{0}}^{\bar{x}-1} \sum_{s=y_{0}}^{\bar{y}-1} \sum_{t=z_{0}}^{\bar{z}-1} \sum_{i=1}^{m} g_{i}(x, y, z) \\
& +c(\bar{x}, \bar{y}, \bar{z}) \\
& \quad \cdot \sum_{r=x_{0}}^{x-1} \sum_{s=y_{0}}^{y-1} \sum_{t=z_{0}}^{z-1} \sum_{i=1}^{m} f_{i}(x, y, z) \psi_{i}\left(\varphi^{-1}(\omega(x, y, z))\right) .
\end{aligned}
$$

Then $k_{1}\left(x_{0}, y, z\right)=c(\bar{x}, \bar{y}, \bar{z}) \sum_{r=x_{0}}^{\bar{x}-1} \sum_{s=y_{0}}^{\bar{y}-1} \sum_{t=z_{0}}^{\bar{z}-1} \sum_{i=1}^{m} g_{i}(x, y$, $z)$ and (51) can be restated as

$$
\begin{aligned}
\omega(x, y, z) \leq a(\bar{x}, \bar{y}, \bar{z}) \exp \left(k_{1}(x, y, z)\right) & \\
& \text { on } \Omega_{(\bar{x}, \bar{y}, \bar{z})} .
\end{aligned}
$$

Along the lines of proof of Theorem 5, we obtain

$$
\begin{aligned}
& \frac{\Delta_{x y}^{2} k_{1}(x, y, z+1)-\Delta_{x y}^{2} k_{1}(x, y, z)}{\psi\left[\varphi^{-1}\left(a(\bar{x}, \bar{y}, \bar{z}) \exp \left(k_{1}(x, y, z)\right)\right)\right]} \\
& \quad \leq c(\bar{x}, \bar{y}, \bar{z}) \sum_{i=1}^{m} f_{i}(x, y, z) .
\end{aligned}
$$

From the above inequality and Theorem 3, we observe that

$$
\begin{aligned}
\Phi_{1}( & \left.k_{1}(x, y, z)\right) \\
\leq & \Phi_{1}\left(k_{1}\left(x_{0}, y, z\right)\right) \\
& +c(\bar{x}, \bar{y}, \bar{z}) \sum_{r=x_{0}}^{x-1} \sum_{s=y_{0}}^{y-1} \sum_{t=z_{0}}^{z-1} \sum_{i=1}^{m} f_{i}(x, y, z) .
\end{aligned}
$$

Now using inequalities (53) and (55) in (49), we get

$$
u(x, y, z) \leq \varphi^{-1}\left\{a(\bar{x}, \bar{y}, \bar{z}) \exp \left[\Phi_{1}^{-1}\left(\Phi_{1}\left(k_{1}\left(x_{0}, y, z\right)\right)+c(\bar{x}, \bar{y}, \bar{z}) \sum_{r=x_{0}}^{x-1} \sum_{s=y_{0}}^{y-1} \sum_{t=z_{0}}^{z-1} \sum_{i=1}^{m} f_{i}(x, y, z)\right)\right]\right\}
$$

Taking $x=\bar{x}, y=\bar{x}$, and $z=\bar{z}$ in the above inequality, since $\bar{x}, \bar{y}$, and $\bar{z}$ are arbitrary, we get the required inequality.

For the special case $\varphi(u)=u^{p}(p>0$ is constant $)$, Corollary 6 gives the following retarded discrete inequality for nonlinear functions.
Corollary 7. Let $u, a, c$ and $f_{i}, g_{i}(i=1,2, \ldots, m)$ be as defined in Theorem 5, and let $\psi$ be as defined in Theorem 3. Suppose that $p>q>0$ are constants. If

$$
u^{p}(x, y, z) \leq a(x, y, z)+c(x, y, z)\left\{\sum_{r=x_{0}}^{x-1} \sum_{s=y_{0}}^{y-1} \sum_{t=z_{0}}^{z-1} u^{p}(r, s, t) \sum_{i=1}^{m}\left[f_{i}(r, s, t) \psi(u(r, s, t))+g_{i}(r, s, t)\right]\right\},
$$

for all $(x, y, z) \in \Omega$, then

$$
\begin{aligned}
& u(x, y, z) \leq a^{1 / p}(x, y, z) \exp \left\{\frac{1}{p}\right. \\
& \cdot \Psi_{1}^{-1}\left(\Psi_{1}\left(k_{1}\left(x_{0}, y, z\right)\right)\right. \\
& \left.\left.+c(x, y, z) \sum_{r=x_{0}}^{x-1} \sum_{s=y_{0}}^{y-1} \sum_{t=z_{0}}^{z-1} \sum_{i=1}^{m} f_{i}(x, y, z)\right)\right\},
\end{aligned}
$$

for all $(x, y, z) \in\left[x_{0}, x_{1}\right] \times\left[y_{0}, y_{1}\right] \times\left[z_{0}, z_{1}\right]$, where $k_{1}\left(x_{0}, y, z\right)$ is defined in Corollary 6 ,

$$
\Psi_{1}(r)=\int_{r_{0}}^{r} \frac{d s}{\psi\left[a^{1 / p} \exp (s / p)\right]}, \quad r \geq r_{0}>0,
$$

$\varphi^{-1}, \Psi_{1}^{-1}$ denote the inverse functions of $\varphi, \Psi_{1}$, and $\left(x_{1}, y_{1}, z_{1}\right) \in \Omega$ is chosen so that

$$
\begin{aligned}
& \left(\Psi_{1}\left(k_{1}\left(x_{0}, y, z\right)\right)\right. \\
& \left.+c(x, y, z) \sum_{r=x_{0}}^{x-1} \sum_{s=y_{0}}^{y-1} \sum_{t=z_{0}}^{z-1} \sum_{i=1}^{m} f_{i}(x, y, z)\right) \\
& \quad \in \operatorname{dom}\left(\Psi_{1}^{-1}\right) .
\end{aligned}
$$

Proof. The proof is followed by an argument similar to that in the proof of Corollary 6 with suitable modification. We omit the details here.

For the special case $\varphi(u)=u^{p}$ and $\phi(u)=u^{q}(p>$ $q>0$ are constants), Theorem 5 gives the following retarded discrete inequality for nonlinear functions. 
Corollary 8. Let $u, a, c$ and $f_{i}, g_{i}(i=1,2, \ldots, m)$ be as defined in Theorem 5, and let $\psi$ be as defined in Theorem 3. Suppose that $p>q>0$ are constants. If

$$
u^{p}(x, y, z) \leq a(x, y, z)+c(x, y, z)\left\{\sum_{r=x_{0}}^{x-1} \sum_{s=y_{0}}^{y-1} \sum_{t=z_{0}}^{z-1} u^{q}(r, s, t) \sum_{i=1}^{m}\left[f_{i}(r, s, t) \psi(u(r, s, t))+g_{i}(r, s, t)\right]\right\},
$$

for all $(x, y, z) \in \Omega$, then

$$
\begin{aligned}
& u(x, y, z) \leq\left\{\Phi _ { 2 } ^ { - 1 } \left(\Phi_{2}\left(k_{2}\left(x_{0}, y, z\right)\right)\right.\right. \\
& \left.\left.\quad+c(x, y, z) \sum_{r=x_{0}}^{x-1} \sum_{s=y_{0}}^{y-1} \sum_{t=z_{0}}^{z-1} \sum_{i=1}^{m} f_{i}(x, y, z)\right)\right\}^{1 /(p-q)},
\end{aligned}
$$

for all $(x, y, z) \in\left[x_{0}, x_{1}\right] \times\left[y_{0}, y_{1}\right] \times\left[z_{0}, z_{1}\right]$, where

$$
\begin{aligned}
& k_{2}\left(x_{0}, y, z\right) \\
& =[a(x, y, z)]^{(p-q) / p} \\
& \quad+\frac{p-q}{p} c(x, y, z) \sum_{r=x_{0}}^{x-1} \sum_{s=y_{0}}^{y-1} \sum_{t=z_{0}}^{z-1} \sum_{i=1}^{m} g_{i}(x, y, z), \\
& \Phi_{2}(r)=\int_{r_{0}}^{r} \frac{d s}{\psi\left(s^{1 /(p-q)}\right)}, \quad r \geq r_{0}>0,
\end{aligned}
$$

$\varphi^{-1}, \Phi_{1}^{-1}$ denote the inverse functions of $\varphi, \Phi_{1}$, and $\left(x_{1}\right.$, $\left.y_{1}, z_{1}\right) \in \Omega$ is chosen so that

$$
\begin{aligned}
& \left(\Phi_{2}\left(k_{2}\left(x_{0}, y, z\right)\right)\right. \\
& \left.+c(x, y, z) \sum_{r=x_{0}}^{x-1} \sum_{s=y_{0}}^{y-1} \sum_{t=z_{0}}^{z-1} \sum_{i=1}^{m} f_{i}(x, y, z)\right) \\
& \quad \in \operatorname{dom}\left(\Phi_{2}^{-1}\right) .
\end{aligned}
$$

Proof. The proof is followed by an argument similar to that in the proof of Theorem 5 with suitable modification. We omit the details here.

Theorems 3 and 5 can easily be applied to generate other useful discrete inequalities in more effective tools of the study of certain partial finite difference and sum-difference equations. For example, we have the following results.

Theorem 9. Let $u, a, c, \varphi, \psi$, and $f$ be as defined in Theorem 3, and let $L: \Omega \times \mathbb{R}_{0} \longrightarrow \mathbb{R}_{0}$ be real-valued function which satisfies the following condition:

$$
\begin{aligned}
0 & \leq L(x, y, z, v)-L(x, y, z, w) \\
& \leq M(x, y, z, w)(v-w),
\end{aligned}
$$

for $x, y, z \in \mathbb{R}_{0}$ and $v \geq w \geq 0$, where $M(x, y, z, w): \Omega \times$ $\mathbb{R}_{0} \longrightarrow \mathbb{R}_{0}$ be real-valued function. If

$$
\begin{gathered}
\varphi(u(x, y, z)) \leq a(x, y, z)+c(x, y, z) \\
\quad \cdot \sum_{r=x_{0}}^{x-1} \sum_{s=y_{0}}^{y-1} \sum_{t=z_{0}}^{z-1} f(r, s, t) L(r, s, t, \psi(u(r, s, t))),
\end{gathered}
$$

for all $(x, y, z) \in \Omega$, then

$$
\begin{aligned}
& u(x, y, z) \leq \varphi^{-1}\left\{\Phi ^ { - 1 } \left(\Phi\left(\omega\left(x_{0}, y, z\right)\right)\right.\right. \\
& \left.\left.\quad+c(x, y, z) \sum_{r=x_{0}}^{x-1} \sum_{s=y_{0}}^{y-1} \sum_{t=z_{0}}^{z-1} f(r, s, t) M(x, y, z)\right)\right\},
\end{aligned}
$$

for all $(x, y, z) \in\left[x_{0}, x_{1}\right] \times\left[y_{0}, y_{1}\right] \times\left[z_{0}, z_{1}\right]$, where $\Phi(r)$ is defined in Theorem 3,

$$
\begin{aligned}
& \omega\left(x_{0}, y, z\right) \\
& =a(x, y, z) \\
& \quad+c(x, y, z) \sum_{r=x_{0}}^{x-1} \sum_{s=y_{0}}^{y-1} \sum_{t=z_{0}}^{z-1} f(r, s, t) L(r, s, t),
\end{aligned}
$$

$\varphi^{-1}, \Phi^{-1}$ denote the inverse functions of $\varphi, \Phi$, and $\left(x_{1}, y_{1}, z_{1}\right) \epsilon$ $\Omega$ is chosen so that

$$
\begin{aligned}
& \left(\Phi\left(\omega\left(x_{0}, y, z\right)\right)\right. \\
& \left.\quad+c(x, y, z) \sum_{r=x_{0}}^{x-1} \sum_{s=y_{0}}^{y-1} \sum_{t=z_{0}}^{z-1} f(r, s, t) M(x, y, z)\right) \\
& \quad \in \operatorname{dom}\left(\Phi^{-1}\right) .
\end{aligned}
$$

Proof. It suffices to consider the case $a(x, y, z)>0$, since the case $a(x, y, z)=0$ can be arrived at by continuity argument. Let us first assume that $a(x, y, z)>0$. Due to the property of $L$, inequality (66) becomes

$$
\begin{gathered}
\varphi(u(x, y, z)) \leq a(x, y, z)+c(x, y, z) \\
\cdot \sum_{r=x_{0}}^{x-1} \sum_{s=y_{0}}^{y-1} \sum_{t=z_{0}}^{z-1} f(r, s, t) \\
\cdot[L(r, s, t)+M(r, s, t) \psi(u(r, s, t))] .
\end{gathered}
$$


Fixing any numbers $\bar{x}, \bar{y}$, and $\bar{z}$ with $x_{0} \leq x \leq \bar{x}, y_{0} \leq y \leq \bar{y}$, and $z_{0} \leq z \leq \bar{z}$, define a positive function $\omega(x, y, z)$ by

$$
\begin{aligned}
& \omega(x, y, z):=a(\bar{x}, \bar{y}, \bar{z})+c(\bar{x}, \bar{y}, \bar{z}) \\
& \quad \cdot \sum_{r=x_{0}}^{\bar{x}-1} \sum_{s=y_{0}}^{\bar{y}-1} \sum_{t=z_{0}}^{\bar{z}-1} f(r, s, t) L(r, s, t)+c(\bar{x}, \bar{y}, \bar{z}) \\
& \quad \cdot \sum_{r=x_{0}}^{x-1} \sum_{s=y_{0}}^{y-1} \sum_{t=z_{0}}^{z-1} f(r, s, t) M(r, s, t) \psi(u(r, s, t))
\end{aligned}
$$

Then $\omega(x, y, z)>0, \omega\left(x_{0}, y, z\right)=a(\bar{x}, \bar{y}, \bar{z})+c(\bar{x}, \bar{y}$, $\bar{z}) \sum_{r=x_{0}}^{\bar{x}-1} \sum_{s=y_{0}}^{\bar{y}-1} \sum_{t=z_{0}}^{\bar{z}-1} f(r, s, t) L(r, s, t)$ and (70) can restated by

$$
u(x, y, z) \leq \varphi^{-1}[\omega(x, y, z)] \quad \text { on } \Omega_{(\bar{x}, \bar{y}, \bar{z})} .
$$

Along the lines of proof of Theorem 3, we obtain

$$
\begin{aligned}
& \frac{\Delta_{x y}^{2} \omega(x, y, z+1)-\Delta_{x y}^{2} \omega(x, y, z)}{\psi\left[\varphi^{-1}(\omega(x, y, z))\right]} \\
& \leq c(\bar{x}, \bar{y}, \bar{z}) f(x, y, z) M(r, s, t) .
\end{aligned}
$$

From the above inequality and Theorem 3, we observe that

$$
\begin{aligned}
& \Phi(\omega(x, y, z)) \\
& \leq \Phi\left(\omega\left(x_{0}, y, z\right)\right) \\
& \quad+c(\bar{x}, \bar{y}, \bar{z}) \sum_{r=x_{0}}^{x-1} \sum_{s=y_{0}}^{y-1} \sum_{t=z_{0}}^{z-1} f(x, y, z) M(r, s, t) .
\end{aligned}
$$

$$
\varphi(u(x, y, z)) \leq a(x, y, z)+c(x, y, z)\left\{\sum_{r=x_{0}}^{x-1} \sum_{s=y_{0}}^{y-1} \sum_{t=z_{0}}^{z-1} \phi(u(r, s, t)) \sum_{i=1}^{m}\left[f_{i}(r, s, t) L(r, s, t, \psi(u(r, s, t)))+g_{i}(r, s, t)\right]\right\}
$$

for all $(x, y, z) \in \Omega$, then

$$
\begin{aligned}
& u(x, y, z) \leq \varphi^{-1}\left\{G ^ { - 1 } \left[\Phi ^ { - 1 } \left(\Phi\left(k_{3}\left(x_{0}, y, z\right)\right)\right.\right.\right. \\
& +c(x, y, z) \\
& \left.\left.\left.\quad \cdot \sum_{r=x_{0}}^{x-1} \sum_{s=y_{0}}^{y-1} \sum_{t=z_{0}}^{z-1} \sum_{i=1}^{m} f_{i}(x, y, z) M(r, s, t)\right)\right]\right\},
\end{aligned}
$$

for all $(x, y, z) \in\left[x_{0}, x_{1}\right] \times\left[y_{0}, y_{1}\right] \times\left[z_{0}, z_{1}\right]$, where $G, \Phi(r)$ are defined in Theorem 5,
Now using inequalities (74) in (72), we get

$$
\begin{aligned}
& u(x, y, z) \leq \varphi^{-1}\left\{\Phi ^ { - 1 } \left(\Phi\left(\omega\left(x_{0}, y, z\right)\right)\right.\right. \\
& \left.\left.\quad+c(\bar{x}, \bar{y}, \bar{z}) \sum_{r=x_{0}}^{x-1} \sum_{s=y_{0}}^{y-1} \sum_{t=z_{0}}^{z-1} f(r, s, t) M(x, y, z)\right)\right\}
\end{aligned}
$$

Taking $x=\bar{x}, y=\bar{x}$, and $z=\bar{z}$ in the above inequality, since $\bar{x}, \bar{y}$, and $\bar{z}$ are arbitrary, we get the required inequality.

Corollary 10. Let $u, a, c, \varphi, \psi$, and $f$ be as defined in Theorem 3, and let $L, M$ be as defined in Theorem 9. If

$$
\begin{aligned}
& \varphi(u(x, y, z)) \leq a(x, y, z)+c(x, y, z) \\
& \cdot \sum_{r=x_{0}}^{x-1} \sum_{s=y_{0}}^{y-1} \sum_{t=z_{0}}^{z-1} f(r, s, t) L(r, s, t, \varphi(u(r, s, t))),
\end{aligned}
$$

for all $(x, y, z) \in \Omega$, then

$$
\begin{gathered}
u(x, y, z) \leq \varphi^{-1}\left\{\omega\left(x_{0}, y, z\right) \exp (c(x, y, z)\right. \\
\left.\left.\cdot \sum_{r=x_{0}}^{x-1} \sum_{s=y_{0}}^{y-1} \sum_{t=z_{0}}^{z-1} f(r, s, t) M(x, y, z)\right)\right\}
\end{gathered}
$$

for all $(x, y, z) \in \Omega$, where $\omega\left(x_{0}, y, z\right)$ is defined in Theorem 9 . Proof. The proof is followed by an argument similar to that in the proof of Corollary 4 and Theorem 9 with suitable modification. We omit the details here.

Theorem 11. Let $\psi$ be as defined in Theorem 3, let $u, a, c, \varphi, \phi$ and $f_{i}, g_{i}(i=1,2, \ldots, m)$ be as defined in Theorem 5, and let $L, M$ be as defined in Theorem 9. If 
Proof. The proof is followed by an argument similar to that in the proof of Theorems 5 and 9 with suitable modification. We omit the details here.

\section{Applications}

In this section, we will show that our results are useful in establishing the boundedness and uniqueness of solutions to certain partial finite difference and sum-difference equations. Consider the partial finite difference equations with the initial boundary conditions:

$$
\Delta_{x y z}^{3} \varphi(u(x, y, z))=F(x, y, z, u(x, y, z))
$$

with

$$
\begin{aligned}
u\left(x_{0}, y, z\right) & =e_{1}(y, z), \\
u\left(x, y_{0}, z\right) & =e_{2}(x, z), \\
u\left(x, y, z_{0}\right) & =e_{3}(x, y), \\
e_{2}\left(x_{0}, z\right) & =e_{3}\left(x_{0}, y\right)=0, \\
e_{1}\left(y_{0}, z\right) & =e_{3}\left(x, y_{0}\right)=0, \\
e_{1}\left(y, z_{0}\right) & =e_{2}\left(x, z_{0}\right)=0 .
\end{aligned}
$$

The following two results deal with the boundedness on the solutions of problem (82).

Proposition 12. Let $F$ be real-valued function such that

$$
\begin{aligned}
& |F(x, y, z, u)| \leq f(x, y, z) \psi(|u|), \\
& \left|\varphi\left(e_{1}(y, z)\right)+\varphi\left(e_{2}(x, z)\right)+\varphi\left(e_{3}(x, y)\right)\right| \\
& \quad \leq a(x, y, z)
\end{aligned}
$$

where $a$ and $f$ are real-valued nonnegative functions defined for $\Omega$ and $a$ is nondecreasing in each variables. Suppose that $\varphi \in C\left(\mathbb{R}_{0}, \mathbb{R}_{0}\right)$ is a strictly increasing function with $\varphi(0)=0$ and $\psi(u)$ is a nondecreasing continuous function with $\psi(u)>0$ for $u \in \mathbb{R}_{+}$. If $u(x, y, z)$ is any solution of problem (82) with the condition (83), then

$$
\begin{gathered}
u(x, y, z) \leq \varphi^{-1}\left\{\Phi^{-1}(\Phi(a(x, y, z))\right. \\
\left.\left.+\sum_{r=x_{0}}^{x-1} \sum_{s=y_{0}}^{y-1} \sum_{t=z_{0}}^{z-1} f(r, s, t)\right)\right\}
\end{gathered}
$$

for all $(x, y, z) \in\left[x_{0}, x_{1}\right] \times\left[y_{0}, y_{1}\right] \times\left[z_{0}, z_{1}\right]$, where $\Phi(r)$ is defined in Theorem 3, $\varphi^{-1}, \Phi^{-1}$ denote the inverse functions of $\varphi$, $\Phi$, and $\left(x_{1}, y_{1}, z_{1}\right) \in \Omega$ is chosen so that

$$
\begin{aligned}
& \left(\Phi(a(x, y, z))+\sum_{r=x_{0}}^{x-1} \sum_{s=y_{0}}^{y-1} \sum_{t=z_{0}}^{z-1} f(r, s, t)\right) \\
& \in \operatorname{dom}\left(\Phi^{-1}\right) .
\end{aligned}
$$

Proof. It is easy to see that the solution $u(x, y, z)$ of problem (82) satisfies equivalent sum-difference equation:

$$
\begin{aligned}
\varphi(u(x, y, z))= & \varphi\left(e_{1}(y, z)\right)+\varphi\left(e_{2}(x, z)\right) \\
& +\varphi\left(e_{3}(x, y)\right) \\
& +\sum_{r=x_{0}}^{x-1} \sum_{s=y_{0}}^{y-1} \sum_{t=z_{0}}^{z-1} F(r, s, t, u(r, s, t)) .
\end{aligned}
$$

From (84) and (87), we have

$$
\begin{aligned}
\varphi(u(x, y, z)) \\
\leq\left|\varphi\left(e_{1}(y, z)\right)+\varphi\left(e_{2}(x, z)\right)+\varphi\left(e_{3}(x, y)\right)\right| \\
\quad+\sum_{r=x_{0}}^{x-1} \sum_{s=y_{0}}^{y-1} \sum_{t=z_{0}}^{z-1}|F(r, s, t, u(r, s, t))| \\
\leq a(x, y, z) \\
\quad+\sum_{r=x_{0}}^{x-1} \sum_{s=y_{0}}^{y-1} \sum_{t=z_{0}}^{z-1} f(x, y, z) \psi(|u(r, s, t)|) .
\end{aligned}
$$

Now, a suitable application of inequality given in Theorem 3 to the above yields the desired result (85).

Proposition 13. Let $F$ be real-valued function such that

$$
\begin{aligned}
& |F(x, y, z, u)| \leq f(x, y, z) L(x, y, z, \psi(|u|)), \\
& \left|\varphi\left(e_{1}(y, z)\right)+\varphi\left(e_{2}(x, z)\right)+\varphi\left(e_{3}(x, y)\right)\right| \\
& \quad \leq a(x, y, z)
\end{aligned}
$$

where $a$ and $f$ are real-valued nonnegative functions defined for $\Omega, a$ is nondecreasing in each variables, and $L: \Omega \times \mathbb{R}_{0} \longrightarrow$ $\mathbb{R}_{0}$ is real-valued function which satisfies the condition

$$
\begin{aligned}
0 & \leq L(x, y, z, v)-L(x, y, z, w) \\
& \leq M(x, y, z, w)(v-w),
\end{aligned}
$$

for $x, y, z \in \mathbb{R}_{0}$ and $v \geq w \geq 0, M(x, y, z, w): \Omega \times \mathbb{R}_{0} \longrightarrow$ $\mathbb{R}_{0}$ be real-valued function. Suppose that $\varphi \in C\left(\mathbb{R}_{0}, \mathbb{R}_{0}\right)$ is a strictly increasing function with $\varphi(0)=0$ and $\psi(u)$ is a nondecreasing continuous function with $\psi(u)>0$ for $u \in \mathbb{R}_{+}$. If $u(x, y, z)$ is any solution of problem (82) with condition (83), then

$$
\begin{gathered}
u(x, y, z) \leq \varphi^{-1}\left\{\Phi ^ { - 1 } \left(\Phi\left(\omega\left(x_{0}, y, z\right)\right)\right.\right. \\
\left.\left.+\sum_{r=x_{0}}^{x-1} \sum_{s=y_{0}}^{y-1} \sum_{t=z_{0}}^{z-1} f(r, s, t) M(x, y, z)\right)\right\},
\end{gathered}
$$

for all $(x, y, z) \in\left[x_{0}, x_{1}\right] \times\left[y_{0}, y_{1}\right] \times\left[z_{0}, z_{1}\right]$, where $\Phi(r)$ is defined in Theorem 3,

$$
\begin{aligned}
\omega\left(x_{0}, y, z\right)= & a(x, y, z) \\
& +\sum_{r=x_{0}}^{x-1} \sum_{s=y_{0}}^{y-1} \sum_{t=z_{0}}^{z-1} f(r, s, t) L(r, s, t),
\end{aligned}
$$


$\varphi^{-1}, \Phi^{-1}$ denote the inverse functions of $\varphi, \Phi$, and $\left(x_{1}, y_{1}, z_{1}\right) \in$ $\Omega$ is chosen so that

$$
\begin{aligned}
& \left(\Phi\left(\omega\left(x_{0}, y, z\right)\right)+\sum_{r=x_{0}}^{x-1} \sum_{s=y_{0}}^{y-1} \sum_{t=z_{0}}^{z-1} f(r, s, t) M(x, y, z)\right) \\
& \quad \in \operatorname{dom}\left(\Phi^{-1}\right) .
\end{aligned}
$$

Proof. The proof is followed by an argument similar to that in the proof of Theorem 3, using Theorem 9 with suitable modification. We omit the details here.

The following theorem deal with the uniqueness on the solutions of problem (82).

Proposition 14. Let F be real-valued function such that

$$
\begin{aligned}
& |F(x, y, z, v)-F(x, y, z, w)| \\
& \quad \leq f(x, y, z)|\varphi(v)-\varphi(w)|
\end{aligned}
$$

where $f$ is real-valued nonnegative function on $\Omega$. Then problem (82) with condition (83) has at most one positive solution on $\Omega$.

Proof. Let $u(x, y, z)$ and $\bar{u}(x, y, z)$ be two solutions of problem (82) on $\Omega$. By (94), we obtain

$$
\begin{aligned}
& \mid \varphi(u(x, y, z))-\varphi(\bar{u}(x, y, z)) \mid \\
& \leq \mid \sum_{r=x_{0}}^{x-1} \sum_{s=y_{0}}^{y-1} \sum_{t=z_{0}}^{z-1} F(r, s, t, u(r, s, t)) \\
&- \sum_{r=x_{0}}^{x-1} \sum_{s=y_{0}}^{y-1} \sum_{t=z_{0}}^{z-1} F(r, s, t, \bar{u}(r, s, t)) \mid \\
& \leq \sum_{r=x_{0}}^{x-1} \sum_{s=y_{0}}^{y-1} \sum_{t=z_{0}}^{z-1} f(r, s, t) \\
& \cdot|\varphi(u(r, s, t))-\varphi(\bar{u}(r, s, t))| .
\end{aligned}
$$

An application of Corollary 4 with suitable modification to function $\varphi^{-1}(|\varphi(u(x, y, z))-\varphi(\bar{u}(x, y, z))|)$ in the last inequality yields

$$
|\varphi(u(x, y, z))-\varphi(\bar{u}(x, y, z))| \leq 0,
$$

for $(x, y, z) \in \Omega$. Hence $u=\bar{u}$ on $\Omega$.

Finally, we investigate the continuous dependence of the solutions of problem (82) on the function $F$ and the boundary data $e_{1}, e_{2}$, and $e_{3}$. For this we consider the following variation of problem (82).

$$
\Delta_{x y z}^{3} \varphi(u(x, y, z))=\bar{F}(x, y, z, u(x, y, z))
$$

with

$$
\begin{aligned}
u\left(x_{0}, y, z\right) & =\bar{e}_{1}(y, z), \\
u\left(x, y_{0}, z\right) & =\bar{e}_{2}(x, z), \\
u\left(x, y, z_{0}\right) & =\bar{e}_{3}(x, y), \\
\bar{e}_{2}\left(x_{0}, z\right) & =\bar{e}_{3}\left(x_{0}, y\right)=0, \\
\bar{e}_{1}(y, z) & =\bar{e}_{3}(x, y)=0, \\
\bar{e}_{1}\left(y, z_{0}\right) & =\bar{e}_{2}\left(x, z_{0}\right)=0 .
\end{aligned}
$$

Proposition 15. Consider problem (82) and problem (97). Assume that

$$
\begin{aligned}
& |F(x, y, z, v)-F(x, y, z, w)| \leq f(x, y, z) \mid \varphi(v) \\
& \quad-\varphi(w) \mid, \\
& \mid \varphi\left(e_{1}(y, z)\right)-\varphi\left(\bar{e}_{1}(y, z)\right)+\varphi\left(e_{2}(x, z)\right) \\
& \quad-\varphi\left(\bar{e}_{2}(x, z)\right)+\varphi\left(e_{3}(x, y)\right)-\varphi\left(\bar{e}_{3}(x, y)\right) \mid \leq \frac{\varepsilon}{2}
\end{aligned}
$$

and

$$
\sum_{r=x_{0}}^{x-1} \sum_{s=y_{0}}^{y-1} \sum_{t=z_{0}}^{z-1}|F(r, s, t, \xi)-\bar{F}(r, s, t, \xi)| \leq \frac{\varepsilon}{2}
$$

where $(x, y, z) \in \Omega$ and $v, w, \xi \in \mathbb{R}$. Then

$$
\begin{array}{r}
|\varphi(u(x, y, z))-\varphi(\bar{u}(x, y, z))| \\
\quad \leq \varepsilon \exp \sum_{r=x_{0}}^{x-1} \sum_{s=y_{0}}^{y-1} \sum_{t=z_{0}}^{z-1} f(r, s, t),
\end{array}
$$

for all $(x, y, z) \in \Omega$. Hence, $\varphi(u)$ depends continuously on $F$, $e_{1}, e_{2}$, and $e_{3}$.

Proof. Let $u(x, t, z)$ and $\bar{u}(x, t, z)$ be solutions of problem (82) and problem (97), respectively. Then $u$ satisfies (87) and $\bar{u}$ satisfies the corresponding equation:

$$
\begin{aligned}
\varphi(\bar{u}(x, y, z))= & \varphi\left(\bar{e}_{1}(y, z)\right)+\varphi\left(\bar{e}_{2}(x, z)\right) \\
& +\varphi\left(\bar{e}_{3}(x, y)\right) \\
& +\sum_{r=x_{0}}^{x-1} \sum_{s=y_{0}}^{y-1} \sum_{t=z_{0}}^{z-1} \bar{F}(r, s, t, \bar{u}(r, s, t)) .
\end{aligned}
$$


Hence, we obtain

$$
\begin{aligned}
& |\varphi(u(x, y, z))-\varphi(\bar{u}(x, y, z))| \leq \mid \varphi\left(e_{1}(y, z)\right) \\
& -\varphi\left(\bar{e}_{1}(y, z)\right)+\varphi\left(e_{2}(x, z)\right)-\varphi\left(\bar{e}_{2}(x, z)\right) \\
& +\varphi\left(e_{3}(x, y)\right)-\varphi\left(\bar{e}_{3}(x, y)\right) \mid \\
& +\mid \sum_{r=x_{0}}^{x-1} \sum_{s=y_{0}}^{y-1} \sum_{t=z_{0}}^{z-1} F(r, s, t, u(r, s, t)) \\
& -\sum_{r=x_{0}}^{x-1} \sum_{s=y_{0}}^{y-1} \sum_{t=z_{0}}^{z-1} \bar{F}(r, s, t, \bar{u}(r, s, t)) \mid \leq \frac{\varepsilon}{2} \\
& +\mid \sum_{r=x_{0}}^{x-1} \sum_{s=y_{0}}^{y-1} \sum_{t=z_{0}}^{z-1} F(r, s, t, u(r, s, t)) \\
& -\sum_{r=x_{0}}^{x-1} \sum_{s=y_{0}}^{y-1} \sum_{t=z_{0}}^{z-1} F(r, s, t, \bar{u}(r, s, t)) \\
& +\mid \sum_{r=x_{0}}^{x-1} \sum_{s=y_{0}}^{y-1} \sum_{t=z_{0}}^{z-1} F(r, s, t, \bar{u}(r, s, t)) \\
& -\sum_{r=x_{0}}^{x-1} \sum_{s=y_{0}}^{y-1} \sum_{t=z_{0}}^{z-1} \bar{F}(r, s, t, \bar{u}(r, s, t)) \mid \leq \varepsilon \\
& +\sum_{r=x_{0}}^{x-1} \sum_{s=y_{0}}^{y-1} \sum_{t=z_{0}}^{z-1} f(r, s, t) \\
& \cdot|\varphi(u(r, s, t))-\varphi(\bar{u}(r, s, t))| \text {. }
\end{aligned}
$$

As an application of Corollary 4 with suitable modification, function $\varphi^{-1}(|\varphi(u(x, y, z))-\varphi(\bar{u}(x, y, z))|)$ in the last inequality yields by assumptions (99)-(101)

$$
\begin{array}{r}
|\varphi(u(x, y, z))-\varphi(\bar{u}(x, y, z))| \\
\leq \varepsilon \exp \sum_{r=x_{0}}^{x-1} \sum_{s=y_{0}}^{y-1} \sum_{t=z_{0}}^{z-1} f(r, s, t),
\end{array}
$$

for all $(x, y, z) \in \Omega$. When restricted to any compact sublattice $\sum_{r=x_{0}}^{x-1} \sum_{s=y_{0}}^{y-1} \sum_{t=z_{0}}^{z-1} f(r, s, t)$ that is bounded,

$$
|\varphi(u(x, y, z))-\varphi(\bar{u}(x, y, z))| \leq \varepsilon \cdot K,
$$

for some $K>0$ for all $(x, y, z)$ in this compact sublattice. Hence, $\varphi(u)$ depends continuously on $F, e_{1}, e_{2}$, and $e_{3}$.

\section{Conclusions}

By using the inequality analyze technique, some new discrete Gronwall-Bellman type inequalities with three independent variables have been obtained. Our main theorems generalize and extend some existing results in the literature. Furthermore, the boundedness and uniqueness of solutions to certain partial finite difference and sum-difference equations have been established by applying our obtained results.

\section{Data Availability}

No data were used to support this study.

\section{Conflicts of Interest}

The authors declare that they have no conflicts of interest.

\section{Authors' Contributions}

All authors contributed equally in this article. They read and approved the final manuscript.

\section{References}

[1] A. Abdeldaim, "Nonlinear retarded integral inequalities of Gronwall-Bellman type and applications," Journal of Mathematical Inequalities, vol. 10, no. 1, pp. 285-299, 2016.

[2] Q. Feng, "Some new generalized Gronwall-Bellman type discrete fractional inequalities," Applied Mathematics and Computation, vol. 259, pp. 403-411, 2015.

[3] S. Salem, "On some systems of two discrete inequalities of Gronwall type," Journal of Mathematical Analysis and Applications, vol. 208, no. 2, pp. 553-566, 1997.

[4] B. Zheng, "Some new discrete fractional inequalities and their applications in fractional difference equations," Journal of Mathematical Inequalities, vol. 9, no. 3, pp. 823-839, 2015.

[5] F. W. Meng and W. N. Li, "On some new nonlinear discrete inequalities and their applications," Journal of Computational and Applied Mathematics, vol. 158, no. 2, pp. 407-417, 2003.

[6] W. Sheng and W. N. Li, "Bounds on certain nonlinear discrete inequalities," Journal of Mathematical Inequalities, vol. 2, no. 2, pp. 279-286, 2008.

[7] W.-S. Cheung, "Some discrete nonlinear inequalities and applications to boundary value problems for difference equations," Journal of Difference Equations and Applications, vol. 10, no. 2, pp. 213-223, 2004.

[8] W.-S. Cheung, Q.-H. Ma, and P. C. Josip, "Some discrete nonlinear inequalities and applications to difference equations," Acta Mathematica Scientia B, vol. 28, no. 2, pp. 417-430, 2008.

[9] W. Cheung and J. Ren, "Discrete non-linear inequalities and applications to boundary value problems," Journal of Mathematical Analysis and Applications, vol. 319, no. 2, pp. 708-724, 2006.

[10] Z. Hou, S. Wu, and W.-S. Wang, "Some retarded difference inequalities of product form and their application," Abstract and Applied Analysis, vol. 2014, Article ID 158375, 6 pages, 2014.

[11] Y. Lin, S. Wu, and W.-S. Wang, "A nonlinear weakly singular retarded henry-gronwall type integral inequality and its application," Journal of Applied Mathematics, vol. 2014, Article ID 218507, 9 pages, 2014.

[12] J. Huang and W.-S. Wang, "Some Volterra-Fredholm type nonlinear inequalities involving four iterated infinite integral and application," Journal of Mathematical Inequalities, vol. 10, no. 4, pp. 1105-1118, 2016.

[13] W. Wang and S. Wu, "Some difference inequalities for iterated sums with applications," Abstract and Applied Analysis, vol. 2013, Article ID 804152, 9 pages, 2013.

[14] B. G. Pachpatte, "On some new inequalities related to certain inequalities in the theory of differential equations," Journal of Mathematical Analysis and Applications, vol. 189, no. 1, pp. 128144, 1995. 
[15] B. G. Pachpatte, "Inequalities applicable in the theory of finite difference equations," Journal of Mathematical Analysis and Applications, vol. 222, no. 2, pp. 438-459, 1998.

[16] B. G. Pachpatte, "On some fundamental integral inequalities and their discrete analogues," Journal of Inequalities in Pure and Applied Mathematics, vol. 2, no. 2, article 15, 2001.

[17] Q. Ma and W. Cheung, "Some new nonlinear difference inequalities and their applications," Journal of Computational and Applied Mathematics, vol. 202, no. 2, pp. 339-351, 2007.

[18] Q. Ma and J. E. Pecarić, "On some new nonlinear discrete inequalities and their applications," Journal of Computational and Applied Mathematics, vol. 11, pp. 215-228, 2009.

[19] Q. H. Ma, "Estimates on some power nonlinear VolterraFredholm type discrete inequalities and their applications," Journal of Computational and Applied Mathematics, vol. 233, no. 9, pp. 2170-2180, 2010.

[20] S. Hussain, S. A. Rehman, H. Khalid, and Q.-H. Ma, "Some new Volterra-Fredholm type discrete inequalities with four iterated infinite sums with applications," Journal of Mathematical Inequalities, vol. 11, no. 2, pp. 495-510, 2017.

[21] B. G. Pachpatte and S. M. Singare, "Discrete generalized Gronwall inequalities in three independent variables," Pacific Journal of Mathematics, vol. 82, no. 1, pp. 197-210, 1979.

[22] S. M. Singare and B. G. Pachpatte, "On certain discrete inequalities of the Wendroff type," Indian Journal of Pure and Applied Mathematics, vol. 11, no. 6, pp. 727-736, 1980. 


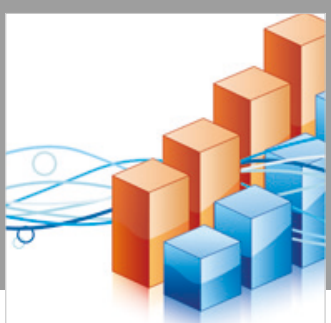

Advances in

Operations Research

\section{-n-m}
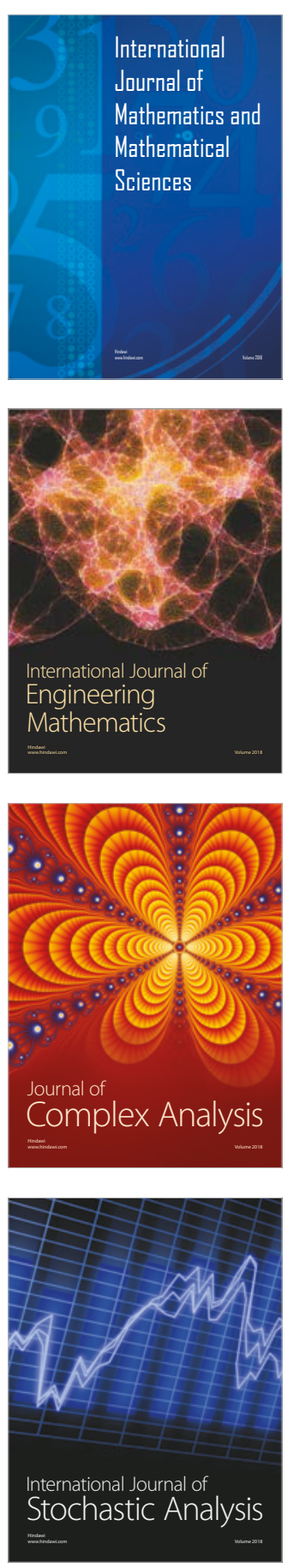
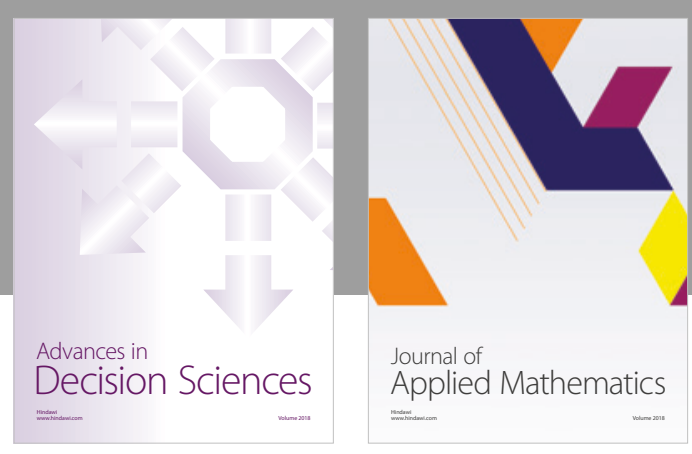

Journal of

Applied Mathematics
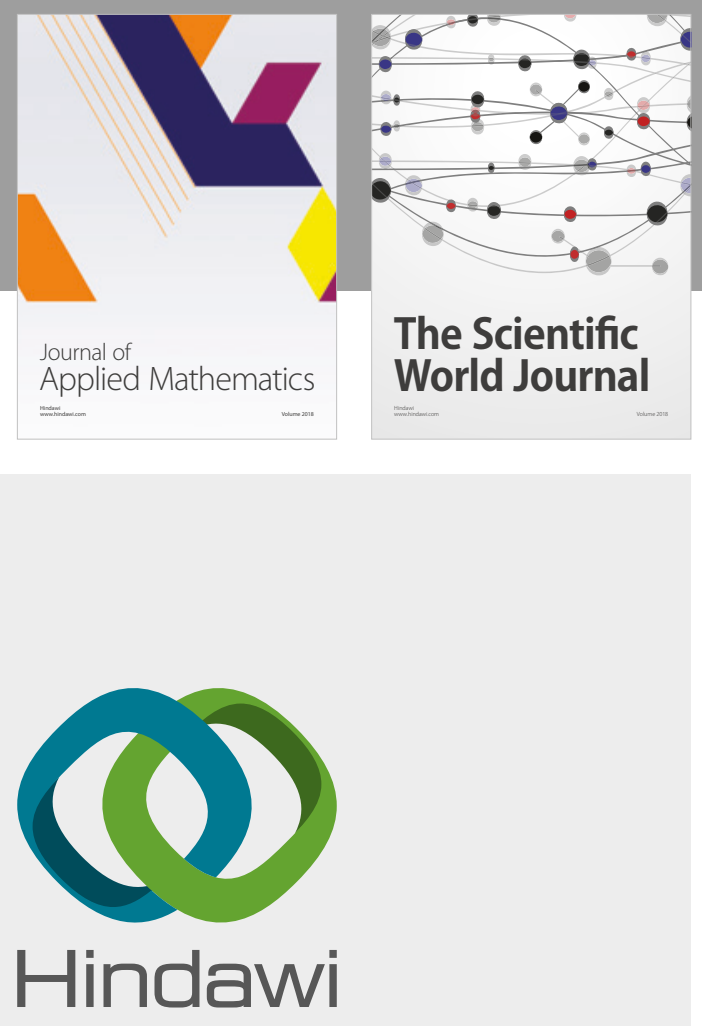

Submit your manuscripts at

www.hindawi.com

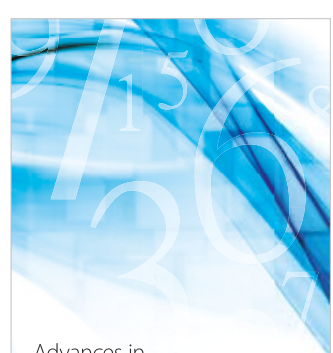

Advances in
Numerical Analysis
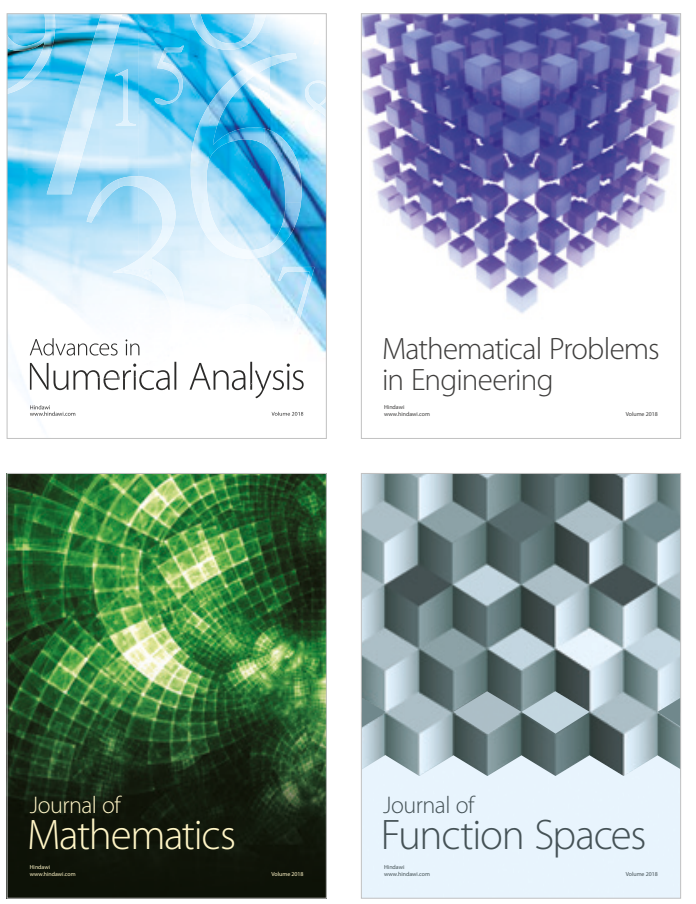

Mathematical Problems in Engineering

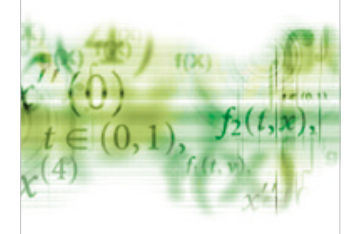

International Journal of

Differential Equations

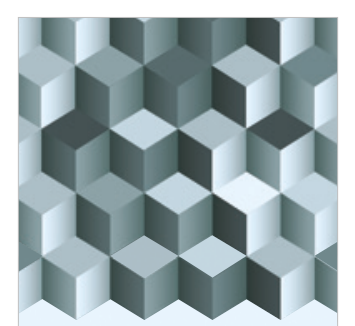

Journal of

Function Spaces

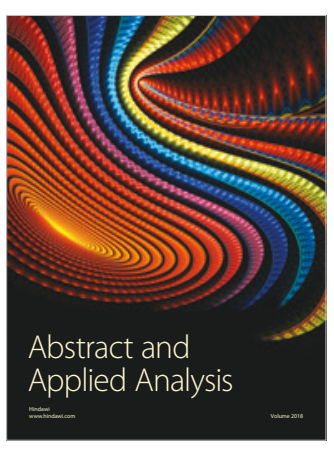

The Scientific

World Journal

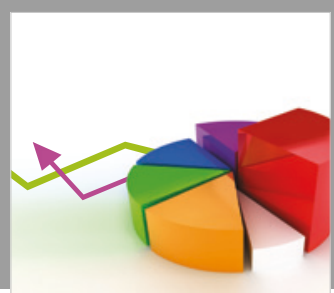

Journal of

Probability and Statistics
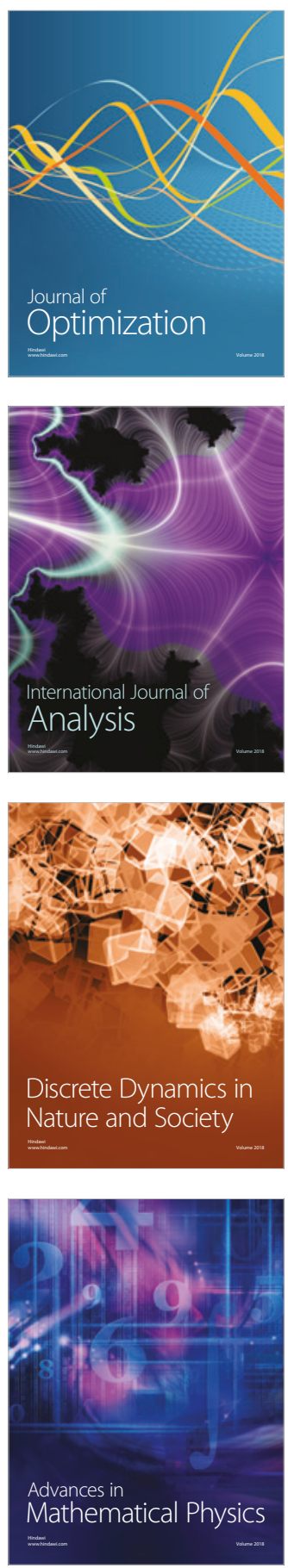